\title{
Rapid particle size measurements used as a proxy to control instant whole milk powder dispersibility
}

\author{
I. Boiarkina ${ }^{1}$ - N. Depree ${ }^{1}$ - W. Yu ${ }^{1}$ - D. I. Wilson ${ }^{2}$. \\ B.R. Young ${ }^{1}$
}

Received: 26 May 2016 /Revised: 24 July 2016 / Accepted: 14 August 2016 /

Published online: 31 August 2016

(C) INRA and Springer-Verlag France 2016

\begin{abstract}
Dispersibility is a key quality variable for instant whole milk powder (IWMP) measured manually using a complex and laborious dissolution method post-production. Consequently, the lack of timely feedback means that the functional quality cannot be controlled in real time. This work proposes the idea of applying a simpler, surrogate measurement that can be implemented in the plant in order to have useful real-time information regarding the quality of the product being produced. This, we term, is a proxy measurement. The functional property dispersibility was used as a case study, with particle size being investigated as a proxy at an industrial IWMP plant. It was found that particle sizing could be used to provide useful information regarding the powder, with the proxy measurement being able to predict in-specification powder $97 \%$ of the time. Although the test was not as effective for predicting out-of-specification results, with a false-positive rate of $50 \%$, the fact that out-of-specification events are rare in the industry setting means that the overall proxy measurement is still between 78 and $87 \%$ accurate and thus useful for predicting the dispersibility quality of the IWMP. Furthermore, these proxy measurements can then be combined with on-line plant information using multivariate techniques to further improve their accuracy and understand how the quality can be controlled by changing the plant processing conditions.
\end{abstract}

Keywords Quality· Dairy· Real time $\cdot$ Proxy measurement $\cdot$ Functional properties

B.R. Young

b.young@auckland.ac.nz

1 Industrial Information and Control Centre, University of Auckland, 2-6 Park Avenue, Grafton, Auckland, New Zealand

2 Industrial Information and Control Centre, Auckland University of Technology, Level 3, Building WU, 46 Wakefield Street, Auckland, New Zealand 


\section{Introduction}

Process analytical technology (PAT) conveys the concept of systems and tools used for achieving end product quality (Munir et al. 2015; Hunter et al. 2012). Historically, much of the focus has been on spectroscopic measurement and the associated modelling of the results, without necessarily closing the feedback loop to ensure this information reaches the process in real time (Munir et al. 2015). However, there are many challenging aspects that need to be addressed in order to be able to close the loop and control the quality during manufacture, when process adjustments can still be made. These key challenges are defining the term 'quality', being able to measure the relevant quality variables, and linking these back to manipulated process variables for control.

What constitutes a quality product is not always straight forward, and this is especially true of food products where a multitude of variables are measured. For example, dairy powders have a number of physical, microbiological, functional and sensory characteristics that are all important in determining the final product quality. A failure in any one of these categories can mean a failure of the product to meet customer specifications, and having individual tests for measuring all possible quality tests online is unlikely to be industrially feasible, if it were even technically possible. This means many tests are carried out post-production, at an external laboratory, once the product quality is fixed.

The consequence of this delayed feedback and the system being sufficiently complex and multi-variable in nature is that it is not immediately obvious what plant inputs affect the different quality outputs. It makes it difficult to correlate cause and effect, especially when data is either missing or incomplete, or the processing and quality data are not time-aligned and stored in separate databases.

Some measurements, such as composition, can be potentially made on-line or at atline, using sophisticated instruments such as near infra-red spectroscopes (NIR) (Hunag et al. 2014, Porep et al. 2015). However, other quality attributes can be very complex and determined by the interaction of both physical and chemical properties. For example, the dissolution properties of dairy powders (e.g. wettability, dispersibility, solubility) are driven by both composition and surface chemistry, as well as physical properties such as the particle size distribution and particle density (Pisecky 2012; Sharma et al. 2012; Schuck 2011). Such tests tend to be highly dependent on the measurement technique and grading scale (Neff and Morris 1967), with industry often using their own modified methods for manufacturing purposes or as per customer requirements.

Thus, this work looks at using an alternate, or proxy, measurement for a complex quality functional test. Such an alternative should be easier to implement and thus lower the barrier to implementation in the plant. Although a proxy measurement would be imperfect (as by definition, it is not the actual test result), it can still be useful. This works towards the concept of real-time quality, where the product quality can be measured or inferred at the time of manufacture, so that timely process adjustments can be made immediately, as needed (Hunter et al. 2012). This work was carried out at an industrial instant whole milk dryer which manufactures a narrow specification commodity product. Although routine measurements of the functional properties is carried out throughout production, this is not done in real time, or hourly. Furthermore, 
on-line measurement of most functional properties is not possible. However, a similar approach could be applied at factories which manufacture a range of powders and where routine off-line hourly measurements of functional properties and particle size are already done, as on-line measurements could replace and lower the manual testing load and provide real-time information.

The 'instant' property in instant whole milk powder (IWMP) is designed to reconstitute rapidly in cold water and is achieved using two additional processing steps over the traditional WMP manufacturing process: agglomeration and the addition of lecithin. The agglomerated powder is obtained by recycling fines to the top of the dryer in order to incorporate them into larger particles, which are more easily penetrable by water. As WMP contains a large hydrophobic fat fraction, a small quantity of the emulsifier lecithin is added in order to make the surface of particles hydrophilic (Pisecky 2012). This improves the dissolution properties, which include wettability, dispersibility and solubility.

One of the instant dissolution properties of IWMP is termed dispersibility which is a measure of how quickly the powder particles break up and dissolve without leaving a residue, and this will be the focus of this study. This case study considers an industrial plant manufacturing IWMP with premium dispersibility performance. This plant failed to consistently meet the dispersibility specification, and due to the lack of immediate feedback, it was not obvious what caused the problem or how to rectify it. Consequently, a proxy measurement of the dispersibility of IWMP using particle size measurements will be used as a case study in order to infer a complex quality variable, dispersibility, which is currently only measured post-production, and cannot be measured on-line. The novelty of this work is in showing that a measurement that can be carried out on-line, particle sizing, can be used to infer a complex quality result, dispersibility, for real-time quality control at an industrial IWMP plant. Only IWMP was tested, as the industrial plant in question produced whole milk powder exclusively. This approach could be applied to other products; however, testing would have to be carried out for each product to re-specify the correlation, and it would be specific to the manufacturing plant.

\section{Materials and methods}

\subsection{Proxy measurement}

Establishing differences between very different powders is comparatively easy, but within the same type of powder, the differences are much more refined. This work was carried out at an industrial plant that produces a narrow specification range of powder, WMP in this case. This means that the variation within some of the physical and/or chemical properties is reduced.

This narrow difference can be used as an advantage. It opens the possibility that only one physical or chemical property drives the main difference in quality within the same type of powder manufactured at the same plant, i.e. that most of the variation can be attributed to a very small number of factors, as the others can be effectively considered constant. And thus, this factor can be used as a proxy measurement for monitoring and controlling a complex quality variable that can otherwise not be measured in real time. 
The key considerations when choosing and testing a proxy measurement depend on the industrial context. Not all quality variables have the same requirements. For some characteristics, both upper and lower specification limits are important, as well as the actual level of the result. An example would be bulk density, where the exact result determines the volume of powder that needs to be packed and shipped, and thus the transportation cost. For other tests, there may only be an upper limit, and the exact result may not be important so long as it does not breach this limit. Many of the dissolution properties of milk powder fall in this context, for example wettability, dispersibility, solubility and the heat stability tests. In these cases, so long as the specification limits are met, there is no direct economic benefit in having a specific result.

If the test level result is considered of secondary value over knowledge, or if the product is meeting the specification, then the next important factors to consider are the false-positive and false-negative rates. Because a proxy test is not the actual test, it should be expected that there will be some error associated with using it as a predictor. However, the false-positive and false-negative rates cannot be too high in order for the test to be useful for decision making. This is especially important when the quality failure events are rare, but nonetheless very costly economically.

For example, Table 1 shows the dispersibility (a dissolution functional property, discussed in Section 2.2) failure rate as a function of bulk density measured at two different points of the spray drying process. The table shows that when both the packer and sifter bulk densities are high, the relative probability of failure increases by approximately 50 times compared to when both the sifter and packer bulk densities are low. However, as the absolute failure rate is still remarkably low, having a high and sifter and packer bulk density cannot be used as proxy, as the majority of the time, $\sim 96 \%$, the powder will still meet specification, even if it is at a higher risk.

Another challenge for a proxy measurement is that it must be repeatable and have good resolution to pick up the differences within the same type of product, as the powder being manufactured at any one industrial plant tends to be fairly consistent. If the proxy test lacks resolution and repeatability, it may completely miss the fine differences in the powder that drive the quality from being in, to being out-ofspecification.

Therefore, a proxy measurement is an alternate measurement of a complex quality variable, such as a functional or sensory characteristic. It has been easy to measure and directly related or proportional to the quality variable of interest, whilst minimising spurious correlations.

Table 1 A table showing the proportion of poor dispersibility results with a high sifter and packer bulk density during industrial spray drying of instant whole milk powder (IWMP)

Dispersibility failure rate

\begin{tabular}{llll}
\hline & & Packer bulk density & \\
& & Low & High \\
Sifter bulk density & Low & $0.08 \%$ & $0.5 \%$ \\
& High & $0.6 \%$ & $3.8 \%$ \\
\hline
\end{tabular}




\subsection{The dispersibility measurement method}

The IDF standard for measuring dispersibility is the ISO 17758| IDF 087 (International Standards Organisation 2014). It involves stirring a specific quantity of milk powder into water at $25^{\circ} \mathrm{C}$ for $20 \mathrm{~s}$ and then passing it through a $150-\mu \mathrm{m}$ mesh to retain any un-dispersed solids. The solids content in the reconstituted milk is then calculated to determine the percentage of the powder that has been dispersed successfully, and the higher the percentage of solids in the final liquid, the better the dispersibility. However, this is not the only method available as noted in (Neff and Morris 1967). For example, the New Zealand Dairy Board method used a different mesh size, of $300 \mu \mathrm{m}$, with a different stirring speed and dispersion time, and looks at the residue content retained by the mesh directly by comparison with a chart (Pisecky 2012). Thus, the precise analysis method can vary and hence can also be product specific. In all cases, the analysis is manually intensive, time consuming, and cannot be automated nor set up on-line easily.

This work used process data obtained directly from an industrial plant, and the actual method used to determine the dispersibility of powder is confidential owing to customer-specific requirements for this production. Notwithstanding, the test is similar to those described above and was classified into three categories: superior dispersing powder, which disperses completely within the test time; off-specification powder, which does not meet customer requirements and; good dispersibility, which is powder that still meets specification but not by a large margin.

\subsection{Using particle size as a proxy measurement for dispersion}

Different types of dairy powders show varying dispersibility performance due to their disparate chemistry. For example, protein powders normally show poor re-hydration and dispersibility characteristics compared with skim and whole milk powders ( $\mathrm{Ji}$ et al. 2016, Fang et al. 2011; Gaiani et al. 2011). Thus when a single type of product is considered, the next dominating factor driving dispersibility performance is the particle size (Pisecky 2012; Schuck 2011; Neff and Morris 1967, Gaiain et al. 2011). The powders tested in this work were all IWMP produced at the same factory, which produces powder within a narrow specification range. Other factors that could have affected the dispersibility were also considered. The particle density was measured and found to be the same between different samples. The whey protein nitrogen index (WPNI) and bulk density results were examined for correlations with the dispersibility and no correlation was found for the WPNI. Although a weak correlation was found with the bulk density, it was not specific enough to be able to be used as a proxy test, as shown in Table 1 and discussed in Section 2.1. Thus, particle sizing, not currently done at the plant, was evaluated.

The recommendations governing the extent of the tails of the particle size distribution for best instant properties of IWMP hypothesised by Pisecky (2012) are as follows:

1. No more than $10-20 \%$ of particles $<125 \mu \mathrm{m}$ in size, (known as the fine fraction)

2. No more than $10 \%$ of particles, $<500 \mu \mathrm{m}$ in size (known as the coarse fraction)

And it is generally agreed that as the percentage of fine particles in the powder increases, the dispersibility worsens (Pisecky 2012; Schuck 2011). Following these 
recommendations, it makes sense to use the particle size as a potential proxy measurement for the dispersibility of IWMP during production. However, it should be noted that these criteria for good performance are fairly loose and it was not clear if this could be transferred to an industrial plant to classify the performance of IWMP, i.e. as a realtime proxy measurement.

A laboratory investigation was launched, and the particle size was first measured at five different IWMP plants in order to see if those used for manufacturing premium grade IWMP had different distributions compared with those used for manufacturing standard powder. If the particle size could not be used for differentiating the powder from the different plants, then it would be unlikely that it could be used to differentiate powder produced from the same plant due to the reduced variation.

\subsection{Particle size measurement}

The particle size measurement depends on the instrument used (Basim and Khalili 2015); therefore, two instruments were tested in order to see if it affected the accuracy of the proxy measurement. Samples of varying dispersibility were collected from an industrial plant and measured using two instruments: (1) the Malvern Mastersizer 2000, a laser diffraction instrument, and (2) sieving to assess the particle size.

When using laser diffraction, approximately $30 \mathrm{~g}$ of milk powder was fed into the feed hopper for the dry dispersion system. The metal ball bearings in the inlet sump were removed, as they caused attrition of the sample. The feed pressure was adjusted to 0.1 bar, and a background reading was taken for $10 \mathrm{~s}$ before the powder feed flow rate was adjusted to the required laser obscuration of 1-5\%. The sample measurement time was set to $20 \mathrm{~s}$. The instrument was further set up with a refractive index of 1.46 and an absorption of 0 .

For sieving, only two sieves were used, giving three size fractions and hence lower resolution, in order to minimise potential powder breakdown during measurement. Thirty grammes of powder was weighed out and mixed with $1 \mathrm{wt} \%$ Syloid 244 FP flow agent from Grace Davison, as IWMP has poor flowability characteristics. The powder was then fractionated using 125- and 355- $\mu \mathrm{m}$ aperture sieves, $200 \mathrm{~mm}$ in diameter, with a vibratory Retsch AS200 sieve shaker for $30 \mathrm{~min}$ at an amplitude of $1.8 \mathrm{~mm}$. The powder remaining on each sieve was weighed and the sieves were then cleaned with detergent and dried before the next analysis.

\section{Results and discussion}

Initially, the particle size was measured across five different plants, and it was found that all of the plants manufactured powder within different particle size ranges. The plant used for manufacturing premium product and achieving the specification consistently manufactured powder with the lowest fines content (particles $<125 \mu \mathrm{m}$ ), varying between 5 and $13 \mathrm{wt} \%$; meanwhile, the plant of interest for implementation of the proxy measurement had 15-24 wt\% fines. Yet another plant that could not be used to manufacture premium grade product had a fines content of approximately $30 \mathrm{wt} \%$. Since only the operating conditions at 
the plants can really be modified (as opposed to the mechanical design of the dryers once built for example), therefore, it is unlikely to be possible to shift the average particle size produced by one plant into the range produced by another; it can only be shifted within a more limited range. This means that the measurement has to be sufficiently sensitive, and repeatable, to pick up meaningful changes in the particle size within powder produced at a single plant.

The particle size of samples taken at the plant of interest measured using sieving and laser diffraction is shown in Fig. 1a, b respectively. Hatched lines have been overlaid to isolate areas where the off-specification product is most concentrated, and these limits are different depending on the measurement. For sieving, in order to isolate offspecification results, it is best to have both an upper fines limit, of $21 \mathrm{wt} \%$, and a lower coarse particle limit, of $16.5 \mathrm{wt} \%$. When using laser diffraction, only one limit was necessary, of maximum $18 \mathrm{vol} \%$ fine particles. The recommendations around the maximum amount of fine particles to meet specification are similar to those discussed in Section 3.2 for powder having good instant properties. However, the exact value varies based on the measurement.

Table 2 shows the false-positive and false-negative rates of using particle size as a proxy for the dispersibility. It can be seen that if the powder is within the particle size recommendations, then it is almost guaranteed to be within specification for dispersibility. There is only a small chance of it not meeting specification, given the samples analysed.

If the powder is predicted to be out-of-specification using the particle size criteria, then there is still roughly a $50 \%$ chance of it being within specification. It would be advantageous to reduce this false-positive rate, i.e. reducing the results that are expected to fail the specification, when they will likely pass the actual test. This is important when considering making changes to the operational conditions, because they may not be necessary if the powder still has a large chance of being within specification. Furthermore, this may divert attention away from other more pertinent plant issues.

Out-of-specification powder occurs infrequently, which means that the proxy test is expected to be accurate $87 \%$ of the time with sieving and $78 \%$ of the time with laser diffraction. Again, the fact that there is a $10 \%$ difference shows that the exact instrument type is important for the measurement and thus should be chosen carefully.

The accuracy of the investigated proxy measurement is sufficiently high to provide useful information regarding the dispersibility of IWMP: if the proxy indicates that the powder being produced is within specification, then there can be confidence in the dryer operating conditions for producing well dispersing powder. Therefore, this approach offers a route for inferring a complex quality variable in real time that otherwise cannot be measured at the time of production. This approach was applied to the same type of powder manufactured from the same plant, where other physiochemical properties were relatively constant. However, this approach can be applied for other products at other plants, with testing to obtain the model for that plant and product combination.

Furthermore, these proxy measurements when done in real time can then be combined with process data using multivariate techniques to both increase the accuracy of the method and understand how the quality is affected by the 

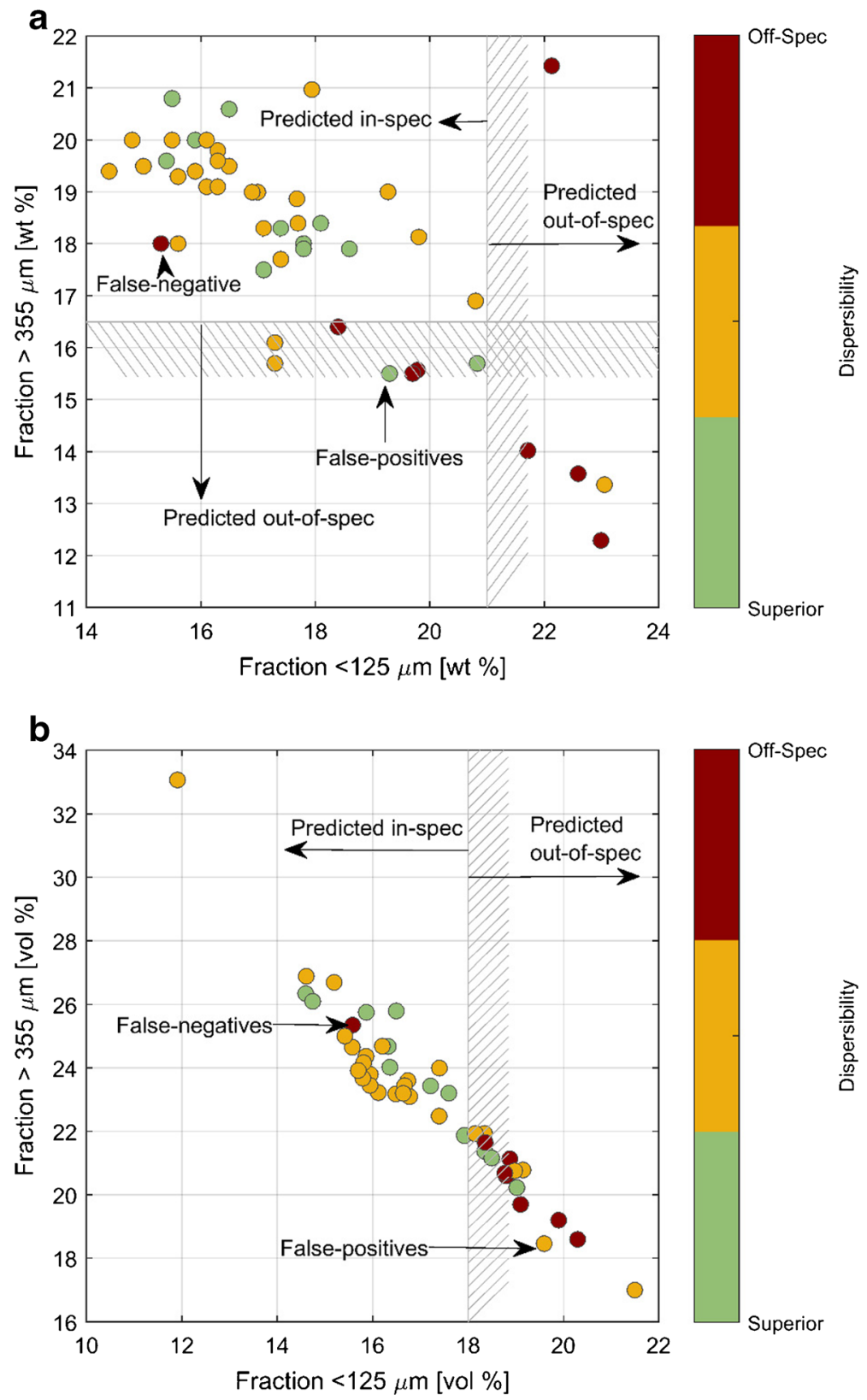

Fig. 1 Suggested criteria for using particle size information as a proxy for dispersibility at the specific industrial plant investigated using a sieving and $\mathbf{b}$ laser diffraction. The overlaid hatched lines isolate most of the off-specification product. Note that the exact limits differ from those given by Pisecky (2012) in Section 3.2

operating conditions. Measurement is only one of the first steps in working towards real-time quality, as one first needs to know if one is achieving the product specifications before knowing whether corrective action needs to be taken. 
Table 2 Estimated false-positive and false-negative rates for using (a) sieving or (b) laser diffraction as a proxy for dispersibility test results (see also Fig. 1)

$\begin{array}{lll}\text { (a) Sieving } & & \\ \text { Dispersibility } & \begin{array}{l}<1 \mathrm{wt} \% \text { fines and }>16.5 \mathrm{wt} \% \\ \text { coarse particles }\end{array} & \begin{array}{c}221 \mathrm{wt} \% \text { fines or } \leq 16.5 \mathrm{wt} \% \\ \text { coarse particles }\end{array} \\ \text { In-specification } & \begin{array}{l}\text { Positive-positive } \\ 97 \%\end{array} & \begin{array}{l}\text { False-positive } \\ 42 \%\end{array} \\ \text { Out-of-specification } & \text { False-negative } & \text { Negative-negative } \\ & 3 \% & 58 \% \\ \text { (b) Laser diffraction } & & \\ \text { Dispersibility } & <18 \text { vol\% fines and }>16.5 \mathrm{wt} \% & \geq 18 \text { vol\% fines and } \leq 16.5 \mathrm{wt} \% \\ & \text { coarse particles } & \text { coarse particles } \\ \text { In-specification } & \text { Positive-positive } & \text { False-positive } \\ & 97 \% & 56 \% \\ \text { Out-of-specification } & \text { False-negative } & \text { Negative-negative } \\ & 3 \% & 44 \%\end{array}$

\section{Conclusions}

This work shows how a proxy measurement (an alternative simpler measurement) can be used to infer a complex product quality variable for application in industry in real time. The measurement of instant whole milk powder (IWMP) dispersibility is only measurable via a manual and laborious test and was used as a case study. Dispersibility measurement is normally carried out post-production at industrial dryers, resulting in no immediate feedback regarding the manufactured powder, and thus difficulty in linking the powder quality to the processing conditions and an inability to control the powder quality in real time. Particle size was tested as a proxy measurement for inferring the dispersibility of IWMP at an industrial plant. It was found that it could be applied in industry to predict the quality of the powder with high confidence, of $97 \%$ given the data, for predicting in-specification product. Although the predictability of out-of-specification product was not as high, $\sim 50 \%$, the low frequency of such events meant that the proxy measurement still had a relatively high accuracy overall. The ability to implement a measurement during production allows for feedback regarding the process to occur in real time, and enables the development of understanding of what processing conditions affect the quality of the product.

Acknowledgments The authors would like to acknowledge the Primary Growth Partnership program from the New Zealand Ministry of Primary Industries for funding the project and would also like to thank the Fonterra staff, specifically James Winchester, Richard Croy, Hong Chen, Nigel Russell and Steve Holroyd, for providing resources and support throughout the project.

\section{References}

Basim GB, Khalili M (2015) Particle size analysis on wide size distribution powders: effect of sampling and characterization technique. Adv Powder Technol 26(1):200-207 
Fang Y, Selomulya C, Ainsworth S, Palmer M, Chen X (2011) On quantifying the dissolution behaviour of milk protein concentrate. Food Hydrocoll 25(3):503-510

Gaiani C, Boyanova P, Hussain R, Murrieta Pazos I, Karam M, Burgain J, Scher J (2011) Morphological descriptors and colour as a tool to better understand rehydration properties of dairy powders. Int Dairy $\mathrm{J}$ 21(7):462-469

Huang Y, Min S, Duan J, Wu L, Li Q (2014) Identification of additive components in powdered milk by NIR imaging methods. Food Chem 145:278-283

Hunter TJ, Russell N, Wilson DI, Young BR, Munir T, Depree N (2012) Developing a real-time quality programme for dairy. Chemeca, 23-36 September, Wellington, New Zealand

International Standards Organisation (2014) ISO 17758| IDF 087 Instant dried milk-determination of the dispersibility and wettability

Ji J, Fitzpatrick J, Cronin K, Maguire P, Zhang H, Miao S (2016) Rehydration behaviours of high protein dairy powders: the influence of agglomeration on wettability, dispersibility and solubility. Food Hydrocoll 58 : 194-203

Munir T, Yu W, Young BR, Wilson DI (2015) The current status of process analytical technologies in the dairy industry. Trends Food Sci Technol 43(2):205-218

Neff E, Morris HAL (1967) Evaluation of reconstitution characteristics of a food powder such as dried milk. Aust J Dairy Technol, vol. September:135-138

Pisecky DIJ (2012) Handbook of milk powder manufacture. GEA Process Engineering A/S, Copenhagen

Porep JU, Kammerer DR, Carle R (2015) On-line application of near infrared (NIR) spectroscopy in food production. Trends Food Sci Technol 46(2):211-230

Schuck P (2011) Milk powder: physical and functional properties of milk powders. In: Encyclopedia of dairy sciences. Academic Press, San Diego, pp. 117-124

Sharma A, Jana AH, Chavan RS (2012) Functionality of milk powders and milk-based powders for end use applications — a review. Compr Rev Food Sci F 11(5):518-528 